\title{
Study on Thermal Effect Model of Laser Irradiating Moving and Rotating Target
}

\author{
Kang Weiwei ${ }^{1, a}$, Qu Zhiyi ${ }^{1, \text { b }}$ and Zhang Donglai ${ }^{1, \text { a }}$ \\ ${ }^{1}$ School of Information Science \& Engineering, Lanzhou University, Lanzhou, China; \\ akangww13@Izu.edu.cn, bquzy@lzu.edu.cn
}

Keywords:Laser irradiating,flying target, heat conduction, irradiating parameters.

\begin{abstract}
A heat conduction model is presented in this paper, with the background of ground-based laser irradiating flying target. Firstly, the encounter scene of laser irradiating target is assumed, and the parameters of laser and flying target are given. Secondly, the calculation models of irradiation radius, power density distribution and convection heat transfer per unit area are derived. Finally, the heat conduction model is established which based on the encounter scene and irradiating parameters. As shown in the example, the heat conduction model can minutely describe the temperature field of metal/explosive structure irradiated by laser.
\end{abstract}

\section{I.Introduction}

The research of laser irradiating cylinder target has got many academic and experimental achievements. C. D. Boley has established a model of laser irradiating cylinder metal/explosive structure, and done an irradiating experiment by solid-state heat-capacity laser (SSCHL) in Lawrence Livermore National Laboratory [1], [2]. Jiao Luguang has numerically simulated the three-dimensional temperature field of metal/explosive irradiating by high energy laser [3], [4].

However, the academic model and experiment in laboratory can't minutely describe the effect of laser irradiating flying cylinder target [5], [6], which should considering the influence of atmosphere propagation and tangential airflow.

The heat conduction model is established in this paper, which includes the factors during laser irradiating flying target, such as the influence of atmosphere propagation and tangential airflow.

This paper is organized as follows. In section II, the encounter scene of laser irradiating is assumed, and the parameters of laser and flying target are given. In sectionIII, the calculation models of irradiation area, power density and convection heat transfer per unit area are derived. In section IV, the heat conduction model is established which based on the encounter scene and irradiating parameters. In section $\mathrm{V}$, based on assuming parameters of laser irradiating flying target, the heat conduction model is analyzed.

\section{II.Encounter Model}

It is a common scene that laser irradiates the target which is flying in the air. The sketch map of encounter scene is shown as Fig.1.
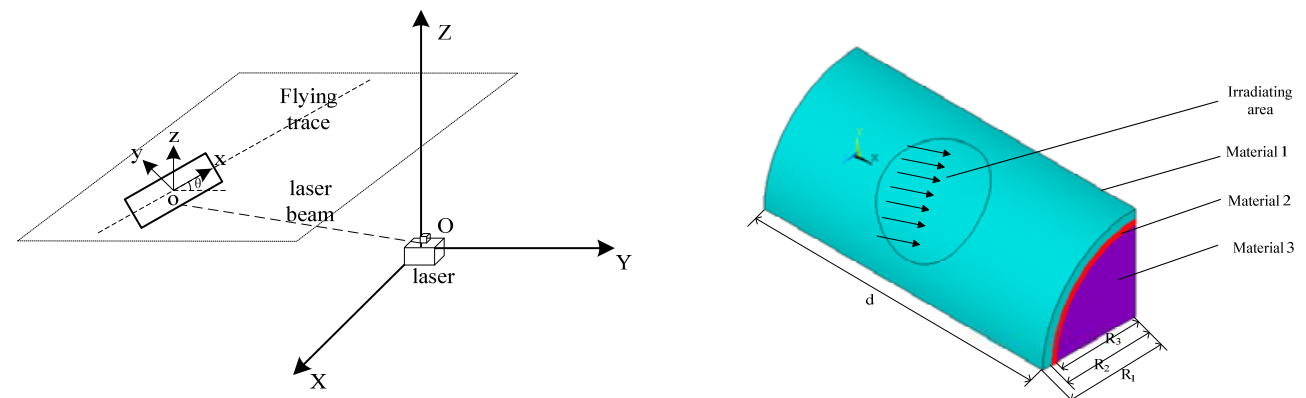

Fig.1 Encounter scene of laser irradiating flying targetFig.2Structure figure of the target About the encounter scene, the paper assumes that as follows[7].

(1)Set the earth as the infinite reference plane; 
(2)To build the global coordinate system as the earth rectangular coordinate system O-XYZ, local coordinate system as target solid rectangular coordinate system o-xyz;

(3)Laser is located in $\mathrm{O}-\mathrm{XYZ}$ coordinate system origin, flying target motion trajectory is located in the plane of the height distance is $\mathrm{H}(\mathrm{H}>0)$, the angle between the flight direction and the OY-axis counterclockwise direction is $\theta(0<\theta<2 \pi)$;

(4)Set initial time of laser irradiation, coordinate of the center point of target is $\left(X_{0}, Y_{0}, H\right)$, target level flight at a constant speed $V_{0}$, axis and orbital overlap, target counterclockwise rotation around its own axis at a constant angular speed $\omega_{0}$;

(5)Laser irradiation position is the cylindrical surface of a cylinder and irradiation center point is located in the center of the laser on the visible part of the cylinder.

The paper defines the target's structure as three layers structure, as shown in Fig.2. The length of the target is a value of $d$. The target includes three layers as metal, insulation and explosive, whose radiuses are $R_{1}, R_{2}$ and $R_{3}$ respectively.

The paper chooses Deuterium Fluoride laser (DF), which is a chemical laser with continuous wave. The wavelength of DF is long enough for laser propagating in the atmosphere. The main parameters of the DF laser are shown in table.1.

Table1 Main parameters of theDF laser

\begin{tabular}{lll}
\hline Parameters & Values & Units \\
\hline Wavelengh $\lambda$ & 3.8 & $\mu \mathrm{m}$ \\
Transmitting Power $P$ & $1 \times 10^{6}$ & $\mathrm{~W}$ \\
Telescope aperture $D_{0}$ & 0.5 & $\mathrm{~m}$ \\
Beam quality factor $\beta$ & 3 & $\circ$ \\
Range of azimuth $\varepsilon$ & 360 & $\circ$ \\
Range of pitch angle $\eta$ & $-20 \sim 85$ & $10^{-3} \mathrm{rad}$ \\
Angle accuracy of tracking $\delta$ & 0.014 & $\mathrm{r}$ \\
\hline
\end{tabular}

The encounter model provides the encounter scene and the parameters of laser irradiating flying target. The encounter model is the basis of the heat conduction model.

\section{III.Irradiating Parameters}

In the encounter scene described above, where laser irradiating flying target level flight at a constant speed.We analyze and solve laser power density distribution oftargetirradiated area domain.

In the $O-X Y Z$ coordinate system, coordinate $\left(X_{\mathrm{F}}, Y_{\mathrm{F}}, Z_{\mathrm{F}}\right)$ offlying target center point can be derived as follows

$$
\left\{\begin{array}{c}
X_{\mathrm{F}}=X_{0}-V_{0} t \cdot \sin \theta \\
Y_{\mathrm{F}}=Y_{0}+V_{0} t \cdot \cos \theta(1) \\
Z_{\mathrm{F}}=H
\end{array}\right.
$$

Laser beam points to the unit vector of the center point of the visible part of the flying target. It can be expressed as laser point to the unit vector of the center point of the flying target approximately, as follows

$$
\overrightarrow{e_{L}}=\frac{\left(X_{\mathrm{F}}, Y_{\mathrm{F}}, Z_{\mathrm{F}}\right)}{\sqrt{{X_{\mathrm{F}}{ }^{2}+Y_{\mathrm{F}}{ }^{2}+Z_{\mathrm{F}}{ }^{2}}^{2}}}(2)
$$

The unit vector of target flying direction can be expressed as

$$
\overrightarrow{e_{n}}=\frac{\left(-V_{0} \cdot \sin \theta, V_{0} \cdot \cos \theta, 0\right)}{V_{0}}(3)
$$

Since it is assumed that direction of the flying target center axis coincides with flying direction, the angle $\alpha(0<\alpha<\pi)$ between the laser beam and the center axis of the cylinder target can be derived.

$\cos \alpha=\overrightarrow{e_{L}} \cdot \overrightarrow{e_{n}}(4)$

Beam diffusion radius $a$ can be calculated by

$a=\mathrm{L} \cdot \Phi(5)$

Where $\mathrm{L}$ is laser transmission distance, which can be calculated by $\sqrt{{X_{\mathrm{F}}}^{2}+{Y_{\mathrm{F}}}^{2}+Z_{\mathrm{F}}{ }^{2}}$; $\Phi$ is beam divergence which is determined by the laser parameters. 
Because of theangle $\alpha$, a circular cross section with radius $\alpha$ of the laser beam is converted into an elliptical cross section with short-axis $\alpha$ and long-axis $\alpha / \sin \alpha$, the projection of which on the cylindrical surface of the target is laser irradiation area. If $\alpha=\pi / 2$, laser beam vertically radiates flying target, the projection of laser irradiation area is a circle.

Since it is assumed that irradiation center point is the center point of the laser on the visible part, the target solid coordinate system o-xyz needs to be solved.

Firstly, matrix $M(\theta)$ is given as follows, which is transformed from coordinate system $O-X Y Z$ to $o-x y z$

$$
M(\theta)=\left[\begin{array}{ccc}
\sin \theta & \cos \theta & 0 \\
\cos \theta & -\sin \theta & 0 \\
0 & 0 & 1
\end{array}\right](6)
$$

Define angle $\phi$ to describe the angle between connection which connects target center point and laser irradiation point with the oy axis. $\phi$ can be described as

$\cos \phi=\frac{[1,0,0]^{*} \times\left([1,0,0]^{*} \times{\overrightarrow{e_{L}}}^{*}\right)}{\left|[1,0,0]^{*} \times\left([1,0,0]^{*} \times{\overrightarrow{e_{L}}}^{*}\right)\right|} \cdot[0,1,0]^{*}(7)$

Where ${\overrightarrow{e_{L}}}^{*}$ is an expression of $\overrightarrow{e_{L}}$ in the coordinate system $o-x y z,{\overrightarrow{e_{L}}}^{*}=M(\theta) \times \overrightarrow{\mathrm{e}_{\mathrm{L}}}$.

The location of spot center point in the coordinate system $o-x y z$ is $(0, R \cdot \cos \phi,-R \cdot \sin \phi)$, where $R$ is the radius of the bottom surface of the cylindrical target.

Assumed that the laser beam intensity follows Gaussian distribution, then the intensity distributing on the transverse face of laser beam is

$I(r)=I_{0} \cdot \exp \left(-2 r^{2} / r_{0}{ }^{2}\right)(8)$

Where $I_{0}$ is intensity of the heat source for laser beam spot center point, $r$ is the distance of any point within the spot to the center point of the spot; $r_{0}$ is the radius of the laser beam spot.

The intensity distribution of transverse face of elliptical laser beam with short-axis $a$ and long-axis $a / \sin \alpha$ is

$$
\mathrm{I}\left(\mathrm{r}_{1}, \mathrm{r}_{2}\right)=\mathrm{I}_{0} \cdot \exp \left(-2 \cdot\left(\frac{\mathrm{r}_{1}{ }^{2}}{\mathrm{a}^{2}}+\frac{\mathrm{r}_{2}{ }^{2}}{\mathrm{a} / \sin \theta}\right)\right)
$$

Where $r_{1}$ and $r_{2}$ are short-axis component and long-axis component of any point within the spot to the center point of spot respectively.

The intensity of the heat source of laser beam spot center $\mathrm{I}_{0}$ can be calculated with average power density integration. And $q$ can be obtained by the laser power $P$ dividing the area of transverse faceof laser beam.

Thus, through determining the position of the center point of the laser irradiation, laser irradiation surface domain, and the heat source intensity of laser spot center, we can determine the power density distribution of laser irradiating flying target level flight at a constant speed.

\section{IV.Heat Conduction Model}

Based on Fourier's Law, in 3D cylindrical coordinates, the heat conduction equation of isotropic material can be described as (10)

$\mathrm{k} \frac{\partial^{2} \mathrm{~T}}{\partial \mathrm{r}^{2}}+\frac{\mathrm{k}}{\mathrm{r}} \frac{\partial \mathrm{T}}{\partial \mathrm{r}}+\frac{\mathrm{k}}{\mathrm{r}^{2}} \frac{\partial^{2} \mathrm{~T}}{\partial \theta^{2}}+\mathrm{k} \frac{\partial^{2} \mathrm{~T}}{\partial \mathrm{z}^{2}}=\rho c \frac{\partial \mathrm{T}}{\partial \mathrm{t}}(10)$

Where $k$ is the thermal conductivity of material; $\rho$ is the density of material; $c$ is the specific heat.

Considering the thermal conductivity and specific heat varying with changes in temperature, (10) is transformed into

$$
K(T)\left(\frac{\partial^{2} T}{\partial r^{2}}+\frac{1}{r} \frac{\partial T}{\partial r}+\frac{1}{r^{2}} \frac{\partial^{2} T}{\partial \theta^{2}}+\frac{\partial^{2} T}{\partial z^{2}}\right)=\rho C(T) \frac{\partial T}{\partial t}(11)
$$

To solve the heat conduction equation, the initial condition and boundary conditions are needed. The initial condition is the temperature of the material at initial time of the heat transfer process.

$\left.T\right|_{t=0}=T_{0}(12)$

Where $T_{0}$ is the temperature of the material at initial time.

The boundary conditions include heat flux caused by laser irradiating and thermal convection caused by airflow as follows 


$$
\left\{\begin{array}{c}
-\left.k \frac{\partial T}{\partial n}\right|_{\Gamma_{1}}=\alpha \cdot I(x, y) \\
-\left.k \frac{\partial T}{\partial n}\right|_{\Gamma_{2}}=q_{c}
\end{array}\right.
$$

Where $\Gamma_{1}$ is the laser irradiating area; $\Gamma_{2}$ is the cylindrical surface of the target; $\alpha$ is the absorption of the target surface.

As shown in fig.2, the values of $k$ and $c$ of three layers is different for different materials. The contacting surfaces among different layers are subject to

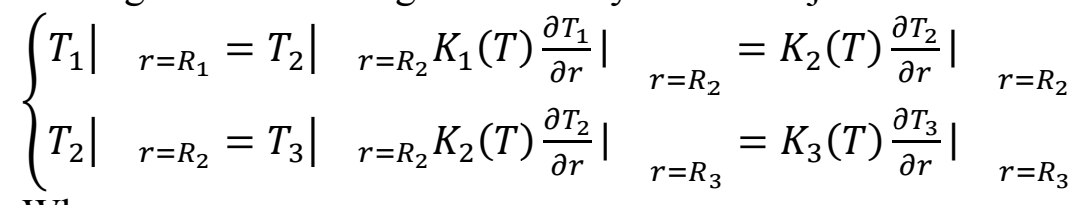

Where

$$
\begin{array}{r}
T(r, \theta, z, t)=\left\{\begin{array}{lr}
T_{1}(r, \theta, z, t) & R_{2} \leq r \leq R_{1} \\
T_{2}(r, \theta, z, t) & R_{3} \leq r \leq R_{2} \\
T_{3}(r, \theta, z, t) & 0 \leq r \leq R_{3}
\end{array}\right. \\
\mathrm{K}(\mathrm{T})=\left\{\begin{array}{lc}
\mathrm{K}_{1}(\mathrm{~T}) & \mathrm{R}_{2} \leq \mathrm{r} \leq \mathrm{R}_{1} \\
\mathrm{~K}_{2}(\mathrm{~T}) & \mathrm{R}_{3} \leq \mathrm{r} \leq \mathrm{R}_{2} \\
\mathrm{~K}_{3}(\mathrm{~T}) & 0 \leq \mathrm{r} \leq \mathrm{R}_{3}
\end{array}\right.
\end{array}
$$

Where $T_{1}(r, \theta, z, t), T_{2}(r, \theta, z, t)$ and $T_{3}(r, \theta, z, t)$ is the temperature functions of metal, insulation and explosive respectively. $K_{1}(T), K_{2}(T)$ and $K_{3}(T)$ are the thermal conductivity of different materials respectively.

The temperature field of the flying target irradiated by laser is given by the heat conduction model, which is described as (11), (12), (13) and (14).

\section{V.Example Analysis}

Parameter setting: the radius of the bottom surface of the cylindrical target is set to $1 \mathrm{~m}$; laser emission power is $1 \times 10^{6} \mathrm{~W}$, beam divergence is $3.27 \times 10^{5} \mathrm{rad}$; energy attenuation coefficient is 0.2 through atmospheric transmission.

In initial time of laser irradiation, set the initial coordinate of flying targetis $[1000,-2000,1000](\mathrm{m})$, and the flying speed is $[-140,350,0](\mathrm{m} / \mathrm{s})$. Laser irradiation time is set to $5 \mathrm{~s}$.

In the rectangular coordinate system $o-x y z$, the angle $\phi$ that between connection which connects target center and laser irradiation with the oy axis changes with time as shown in Fig.3.

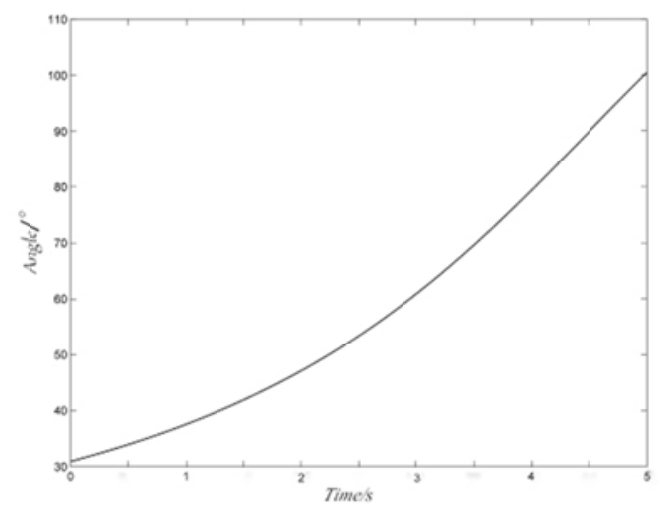

Fig.3 Value of angle $\phi$ varying as time Fig4 Long-axis and short-axis of laser beam

According to the angle $\phi$, the position of laser irradiation center point is determined, i.e. $(0, R$. $\cos \phi,-R \cdot \sin \phi)$.

It is seen from the solution results that in the encounter scene, where laser irradiating flying target level flight at a constant speed, the angle $\phi$ gradually increases with target flying. While the irradiation 
center moves toward the bottom of the target gradually. Whent $=4.5 \mathrm{~s}$, the $\phi=90^{\circ}$ and the coordinate of the target center is $(0,0,-R)$, which is located at the bottom of the target.

We can further figure out the change of the long-axis and short-axis of transverse face of elliptical laser beam with time, which is shown in Fig.4.

With the target flying, the angle $a$ between laser beam and the center axis of the cylindrical target decreases with time. When $t<4.14 \mathrm{~s}$, with the increase of irradiation time, the long-axis and short-axis of transverse face of elliptical laser beam decreases gradually; When $t=4.14 \mathrm{~s}$, the angle $\alpha=90^{\circ}$, the laser beam vertically irradiates flying target, the laser beam transverse face is circular, and the radius of the irradiation is $3.3 \mathrm{~cm}$; When $t>4.14 \mathrm{~s}$, with the increase of irradiation time, the long-axis and short-axis of transverse face of elliptical laser beam increase gradually.

The power density distribution of laser irradiation area can be calculated by analyzing. The power density distribution is given at initial time and the laser irradiation at vertical time.

When $t=0 \mathrm{~s}$, the average power density of laser irradiation target $q$ is $3.98 \times 10^{3} \mathrm{~W} / \mathrm{cm}^{2}$, the heat source intensity of laser spot center is $1.84 \times 10^{4} \mathrm{~W} / \mathrm{cm}^{2}$, the short-axis of elliptical Gaussian beam is $8 \mathrm{~cm}$ and the long-axis is $19.2 \mathrm{~cm}$. The power density distributing of the irradiation area on the cylindrical surface is as depicted in Fig.5.

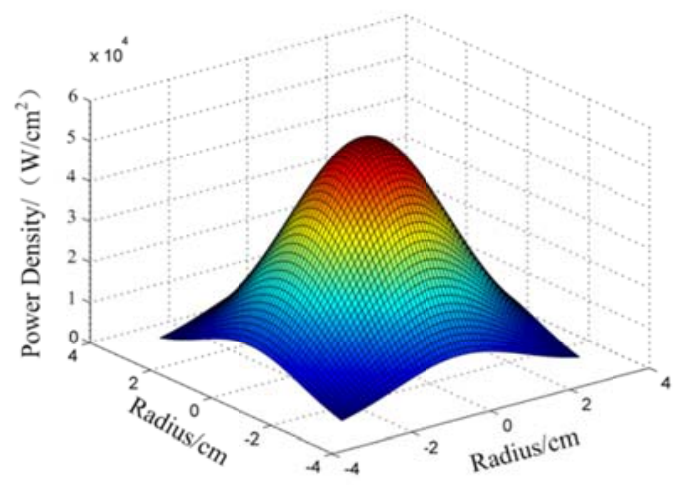

Fig.5Power destiny distributing at $t=0 \mathrm{sFig}$.6Power destiny distributing at $t=4.14 \mathrm{~s}$

When $t=4.14 \mathrm{~s}$, we can figure out that, the average power density of laser irradiation target $q$ is $2.34 \times 10^{4} \mathrm{~W} / \mathrm{cm}^{2}$, the heat source intensity of laser spot center $I_{0}=5.4 \times 10^{5} \mathrm{~W} / \mathrm{cm}^{2}$, the radius of circular Gaussian beam is $3.3 \mathrm{~cm}$. The power density distributing of the irradiation area on the cylindrical surface is as depicted in Fig.6.

\section{VI.Conclusion}

A thermaleffectmodel is proposed that describes the temperature field of flying target irradiated by laser.The model considers the factors of the atmospherepropagation and tangential airflow. The calculating results of the model provide a basis for research of the effect of laser irradiating flying target.

\section{References}

[1] C. D. Boley and A. M. Rubenchik. ModelingAntimortar Lethality by a Solid-State Heat-Capacity Laser. J. Directed Engergy. 2006(2), pp: 97-106.

[2] C. D.Boley, S. Fochs, J.Parker, M. Rotter, ARubenchik and R. Yanmamoto. A Directed Energy System for Defeat of Improvised Explosive Devices and Landmines. Seventh International Symposium on Technologyand the Mine Problem. Monterey, CA, United States, May 2006, pp: 58-69. 
[3] Jiao Luguang and Zhao Guomin. Numerical Simulation of Three-dimensional Temperature Field of Metal/explosive Irradiated by High Energy Laser. Infrared andLaser Engineering. 2001, 40(9), pp: $1715 \sim 1719$.

[4] Jiao Lugang. Effects ofLaser Irradiation to Metal/Explosive.Graduate School of National University of Defense Technology,2008.

[5] Li Haiyan, Hu Yunan and Liu Xudong.Energy estimation method for laser disturbing photoelectrical detectors in remote distance.Infrared and Laser Engineering. 2010, 39(6), pp: 1038-1043.

[6] Hong Yanji. Physics in Direct Energy Technilogy. Beijing: National Defense Industry Press. 2011.

[7]Zhang Donglai, Li Xiaojiang, Yang Yewei. “Analytic Solution of Laser Irradiation Parameters for the Rolling Flying Target”. Chinese Journal of Lasers.2014. 